LBL- -29946

DE92 000861

\title{
Commissioning Experiences of the ALS Booster Synchrotron
}

\author{
Charles Kim \\ Accelerator Fusion Research Division \\ Lawrence Berkeley Laboratory \\ University of California \\ Berkeley, CA 94720
}

May 1991

Presented at the Particle Accelerator Conference, 6-9 May 1991,

San Francisco, CA

This work was supported by the Director, Office of Energy Research, Office of Basic Energy Sciences, Material Sciences Division, U.S. Department of Energy, under Contract No. DE-ACO376SF00098. 


\title{
Commissioning Experiences of the ALS Booster Synchrotron
}

\author{
Charles H. Kim \\ Lawrence Berkeley Laboratory \\ M/S 80-101 \\ Berkeley, CA 94720
}

\section{Abstract}

Installation of the ALS booster synchrotron proper was completed on April 30, 1991, and commissioning has just begun. Circulating beam around the booster was observed on the first day of operation, May 3,1991 . The beam was visible for about 400 turns. In this paper we describe the status and commissioning experience of the $1.5-\mathrm{GeV}$ electron synchrotron accelerator.

\section{Introduction}

Construction of the Advanced Light Source (ALS), a third-generation synchrotron radiation sourc for the $L Y$ and soft X-ray region at LBL [1], entered a new phase recently with beneficial occupancy of the building and with the start of commissioning of the booster. The ALS injector consists of a $50-\mathrm{MeV}$ S-band linac and a 1.5-GeV booster synchrotron [2]. Commissioning of the linac is described else where in these proceedings [3].

The booster consists of 24 dipole and 32 quadrupole magnets in a missing-magnet FODO-lattice configuration with a superperiodicity of 4 . The booster also has 20 sextupole magnets for chromaticity corrections and 32 corrector magnets for orbit corrections. The syschrotron was designed to accelerate a current of $20 \mathrm{~mA}(5 \mathrm{nC})$ in the multibunch mode and $4 \mathrm{~mA}$ $(1 \mathrm{nC})$ in the single-bunch mode. Full cnergy injection into the $1.5-\mathrm{GeV}$ storage ring is expected to take few minutes. Lattice parameters are summarized in Table 1.

Table 1

Booster Lattice Parameters

\begin{tabular}{|l|c|}
\hline Energy & $1.5 \mathrm{GeV}$ \\
Circumference & $75 \mathrm{~m}$ \\
Rf Frequency & $499.7 \mathrm{MHz}$ \\
Harmonic Number & 125 \\
Revolution Frequency & $3.997 \mathrm{MiHz}$ \\
Radiation Loss & $112 \mathrm{keV}$ \\
Natural Energy Spread & $0.064 \%$ \\
Radiation Damping [msec] & \\
Horizontal & 6.68 \\
Vertical & 0.72 \\
Energy & 3.37 \\
\hline
\end{tabular}

\section{Fabrication}

The prototype dipole and quadrupole magnets have been fabricated, measured for multipole errors, and qualified for production $[4,5,6]$. The production magnets have been measured for their magnet-to-magnet reproducibility [7]. These errors were controlled such that the dynamic aperture is larger than the vacuum chamber aperture [8].
The booster vacuum chamber is made of stainless-steel pipes, $30 \mathrm{~mm}$ in radius and $1 \mathrm{~mm}$ in wall thickness. Pipes in the dipole magnets were flattened to $\pm 20 \mathrm{~mm}$ to fit the gap and bent to the proper radius. Average booster vacuum pressure reached lower than $5 \times 10^{-8}$ Torr after a few days of pumping.

Eddie currents, induced on the vacuum chamber wall when the dipole magnetic fields are ramped, generate defocusing sextupole fields whose magnitudes are proportional to dB/dt. The sextupole fields reduce the dynamic aperture severely if the repetition rate is higher than $4 \mathrm{~Hz}$ [8]. The repetition rate of the booster is limited by the dipole power supply to $1 \mathrm{~Hz}$.

\section{Installation}

All magnets, vacuum chambers, and instrumentation in the bends were preassembled on 12 girders. When placing the dipoles, the magnet with the most positive error and most negative error were paired and placed adjacent to each other to minimize the closed-orbit distortion. Each magnet was then prealigned with respect to the girder on which it was mounted and the girders were transported to the accelerator building and lowered into the booster cave. Straight sections (injection, extraction, diagnostic, and if straight sections) were installed subsequently in the tunnel. After utility connections were made, the magnets were aligned again with respect to the girder and the girder with respect to the ALS coordinate system. We have finished aligning most of the girders at the present time with alignment errors $< \pm 300 \mu \mathrm{m}$, which is better than the specifications ( $300 \mu \mathrm{m} \mathrm{rms)} \mathrm{[9].}$

Dipole magnets are connected in series to an SCRswitched power supply [10]. (We used a small dc power supply for the dipoles in the initial injection study.) The focusing (and likewise the defocusing) quadrupoles are connected in series to a power supply-which tracks the dipolemagnet excitation current. Sextupole- and corrector-magnet power supplies also track the dipole current. Current tracking alone does not insure that the magnetic fields will track the dipole field because of the nonlinearities such as the residual fields and core saturation. Therefore, provisions are made in the control system to add small corrections of arbilrary shape to all magnet power supplies that track the dipole power supply.

\section{Operation}

The booster is controlled by the ALS control system [11] using intelligent local controllers (ILCs), which are highly distributed and centrally connected to collector micro-modules via fiber-optical links. Operator interface is via a number of personal computers (six 486/PCs at present) using mostly commercially available software and development tools. Applications are being developed jointly by the ALS control systems group and the accelerator systems group. 
Because of the construction and installation activities during normal working hours, injector commissioning activities have been limited to the evenings.

\section{Injection Studies}

Parameters of the injected beam are summarized in Table 2. Linac operation is very reliable at these operating conditions. Energy spread is mainly due to the energy droop in the subsequent bunches and is high because (1) the beam loading compensation has not been implemented, and (2) the bunching system has not been fully optimized yet. We have a beam collimator for energy selection in the linac-to-booster transfer line. The collimawr was left wide open in the present experiment for the initial tuning purpose.

Table 2

Injected Beam Parameters

\begin{tabular}{|l|c|}
\hline Beam energy & $45 \mathrm{MeV}$ \\
Charge per bunch & $0.1 \mathrm{nC}$ \\
Separation between bunches & $8 \mathrm{~ns}$ \\
Number of bunches & 20 pulses \\
Energy spread & $\pm 2 \%$ \\
Repetition rate & $1 \mathrm{~Hz}$ \\
\hline
\end{tabular}

The booster lattice was set up according to the "nominal tune" column in Table 3.

Table 3

Two Typical Operating Points of the Booster

\begin{tabular}{|l|c|c|}
\hline & $\begin{array}{c}\text { nominal } \\
\text { tune }\end{array}$ & $\begin{array}{c}\text { low } \\
\text { tune }\end{array}$ \\
\hline Betatron Tune & & \\
Horizontal & 6.264 & 5.764 \\
Vertical & 2.789 & 2.480 \\
Momentum Compaction & 0.0408 & 0.0466 \\
Chromaticity & -11.2 & -8.31 \\
$\quad$ Horizontal & -4.79 & -4.69 \\
$\quad$ Vertical & 0.15 & 0.18 \\
Emittance [nm, unnorm] & & \\
Quadrupole $k L[1 / \mathrm{m}]$ & 0.830 & 0.787 \\
Focusing & 0.504 & 0.471 \\
$\quad$ Defocusing & & \\
Sextupole $k L\left[1 / \mathrm{m}^{2}\right]$ & 0.971 & 0.867 \\
$\quad$ Focusing & 1.183 & 0.989 \\
\hline Defocusing & &
\end{tabular}

for quadrupoles $k=(d B / d x) / \mid B \rho]$

for sextupoles $k=\left(d^{2} B / d x^{2}\right) / 2[B \rho]$

Injection from the $50-\mathrm{MeV}$ linear accelerator is via a fast kicker magnet [12] utilizing the well-established single-turn, on-axis injection technique. The fast kicker consists of $(w)$ $25-\mathrm{cm}$ modules and provides a 60-mrad kick to the injected beam onto the booster beam axes. The injection kicker has a 150-nsec flat top, with rise and fall times of about $100 \mathrm{nsec}$. Each module has a pulse flatness of $\pm 0.5 \%$. When fully commissioned, the entire $150 \mathrm{nsec}$ period will have a flatness of $< \pm 0.5 \%$.

Booster instrumentation includes 5 TV monitor stations, 32 beam-position monitors (BPMs), travelling wave electrodes (TWEs) [13], and one beam-intensity monitor (DCCT). A
TV monitor station consists of a Chromox 6 fluorescent screen and a CCD camera. A BPM utilizes 4 button monitors and is similar to the ALS storage ring BPM system [14].

We first observed the injected beam on the fluorescent screen located on the down-stream side $1005 \mathrm{~mm}$ from the center of the kicker magnet where the dispersion is still very small. (The lattice functions at the screen are: horizontal $\alpha=$ 3.93; $\beta=8.45 \mathrm{~m} ; \eta=0.12 \mathrm{~m}$; vertical $\alpha=-1.53 ; \beta=3.37$ $\mathrm{m}$.) The beam centroid moved about $6 \mathrm{~mm}$ as expected when the kicker amplitude was varied by about $10 \%$.

Next, we observed the beam with one of the BPMs located $5586 \mathrm{~mm}$ from the center of the kicker magnet. This BPM is located immediately after the focusing quadrupole in which the dispersion function is very large $(\mathrm{z}=5.3425 \mathrm{~m}$; horizontal $\alpha=4.23 ; \beta=9.17 \mathrm{~m} ; \eta=1.05 \mathrm{~m} ;$ vertical $\alpha=1.98 ; \beta$ $=6.07 \mathrm{~m}$.) We observed that more than half of the 20 bunches were already lost at this location.

Finally, we observed the circulating beam using one of the TWEs in the third quadrant. Figure 1 shows that only the first 3 bunches survived up to the third quadrant. It also shows that about half of the beam intensity was lost by the next turn.

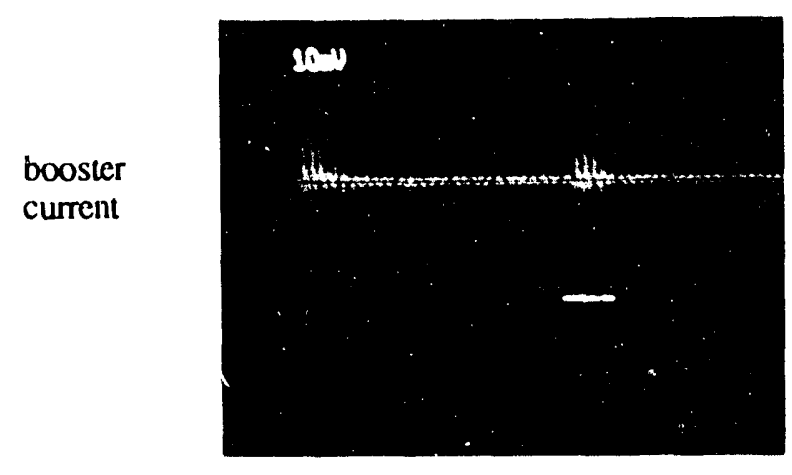

$l(50 \mathrm{nsec} / \mathrm{div})$

Figure 1. TWE signal showing electron bunches for the first two turns. [XBB 915-3445]

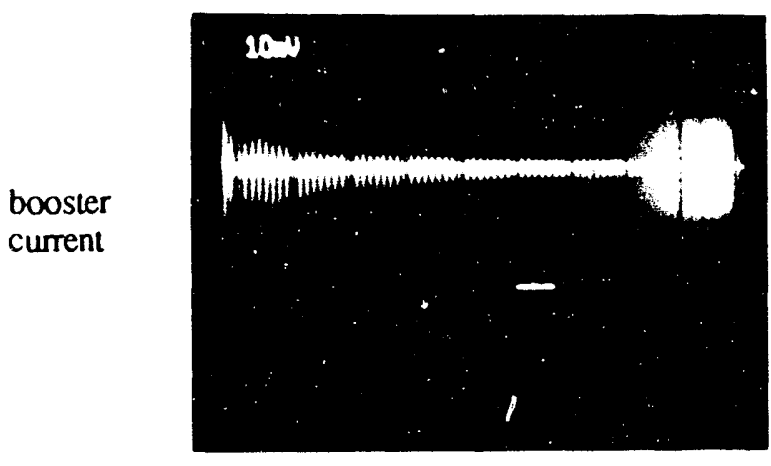

$$
\mathfrak{l}(2 \mu \sec / \text { div })
$$

Figure 2. TWE signal showing electron bunches for the first $20 \mu \mathrm{sec}$. [XBB 915-3446] 
Figure 2 shows the TWE signal for the first $20 \mu \mathrm{sec}$. It shows that the rapid beam loss during the first few turns is followed by a slower beam decay. Circulating beam was visible for about 400 turns.

\section{Summary and Discussions}

We were actually surprised to observe that the beam survived as iong as it did with neither steering magnets nor the if cavity energized. Rapid beam loss was expected from the beginning because of (1) the large energy spread in the injected beam; (2) large closed-orbit distortion; and (3) imperfect flatness of the injection kicker wave form. Our beam stay-clear specifications are summarized in Table 4.

Table 4

Beam Stay-Clear Specifications [mm rms]

\begin{tabular}{|l|c|c|}
\hline Beam Size & At 50 MeV & At 1.5 GeV \\
Dispersion & 3.6 & 1.3 \\
Energy Spread [\%] & 3.5 & 0.7 \\
Closed Orbit distortion & $(0.3)$ & $(0.06)$ \\
before correction & 6.0 & 6.0 \\
after correction§ & 0.3 & 0.3 \\
$\begin{array}{l}\text { Quadratic Sum } \\
\text { before correction }\end{array}$ & 7.8 & 6.2 \\
after correction & 5.0 & 1.5 \\
Pipe Size & \pm 30.0 & \pm 30.0 \\
\hline
\end{tabular}

$\S$ requires corrector strength of $0.5 \mathrm{mad} \mathrm{ms}$

We expect the specifications will be met in the near future as we finish aligning the rest of the magnets, execute beam loading compensation, tune the linac for a smaller energy spread, and flatten the kicker pulse. We will be able to implement orbit corrections and tune fitting as soon as the $\mathrm{rf}$ system and the timing system come on line.

Components necessary for beam acceleration are to be in place by late June. The extraction system is expected to be on line in September, and the booster-to-storage-ring transfer line in October 1991. Storage ring injection is expected to occur in Spring 1992.

\section{Acknowledgements}

Material presented in this paper is the result of a cooperative effort by many groups of the ALS project team, whose personal commitment and unselfish cooperation are gratefully acknowledged. Only some of the groups are listed below: Magnet design and fabrication efforts led by Jack Tanabe, vacuum system by Kurt Kennedy, mechanical installation by Tom Henderson, electrical installation by Ivan Lutz, survey and alignment by Ted Lauritzen, beam diagnostics by Jim Hinkson, injection-extraction mechanical system by John Milburn, kicker and fast magnets by George Gabor, power supply by Terry Jackson, controls group by Steve Magyary, analysis of the magnet measurements and survey and alignment data by Rod Keller. Special thanks are due to Alan
Jackson for his guidance and encouragement and ALS operations group for the long evening shifts.

This work was supported by the Office of Energy Research, Office of Basic Energy Sciences, US Department of Energy, under Contract No. DE-AC03-76SF00098.

\section{References}

1. A. Jackson, "The Advanced Light Source: Status Report," in these proceedings.

2. F. Selph, A. Jackson, and M. S. Zisman, "Injector System Design," Proc. of the 1987 IEEE Particle Accelerator Conference, Washington, D. C., p. 446.

3 F. Selph and D. Mossoletti, "Operating Experience with ALS Linac," in these proceedings

4. J. Tanabe et al., "Fabrication and Test of Prototype Ring Magnets for the ALS," Proceedings of the 1989 IEEE Particle Accelerator Conference, March 20-23, 1989, Chicago, IL p. 566

5. R. Keller et al., "Magnetic Properties of the ALS Booster Synchrotron Engineering Model Magnets," Proceedings of the 1989 IEEE Particle Accelerator Conference, March 20-23, 1989, Chicago, Il p. 1966.

6. M. I. Green, E. Hoyer, R. Keller, and D. H. Nelson, "AC Magnetic Measurements of the ALS Booster Dipole Engineering Model Magnets," Proceedings of the 1989 IEEE Particle Accelerator Conference, March 20-23, 1989, Chicago, IL p. 1969.

7. R. Keller, "Magnetic Data Analysis for the ALS Multipole Magnets," in these proceedings.

8. C. Kim and H. Nishimura, "Dynamic Aperture for the ALS Booster Synchrotron," Proceedings of the 1989 IEEE Particle Accelerator Conference, March 20-23, 1989, Chicago, IL p. 1328.

9. R. Keller, C. Kim, and H. Nishimura, "Alignment Tolerances for the ALS Storage Ring and Booster Synchrotron," LBL ALS Report LSAP-70 (1989)

10. L. T. Jackson and I. C. Lutz, "Magnet Power Supplies for the Advanced Light Source," Proceedings of the 1989 IEEE Particle Accelerator Conference, March 20-23, 1989, Chicago, IL p. 74.

11. S. Magyary et al., "Acvanced Light Source Control System," Proceedings of the 1989 IEEE Particle Accelerator Conference, March 20-23, 1989, Chicago, IL p. 74.

12. G. Gabor and F. Voelker, "Kicker Magnets for ALS" Proceedings of the 1989 IEEE Particle Accelerator Conference, March 20-23, 1989, Chicago, IL p 405.

13. J. H. Hinkson and K. Rex, "A Wide band slot coupled beam sensing electrode for AJS," in these procec.dings.

14. J. Hinkson, J. Johnston, and I. Ko, "ALS Beam Position Monitor," Proceedings of the 1989 IEEE Particle Accelerator Conference, March 20-23, 1989, Chicago, IL, p. 1507. 

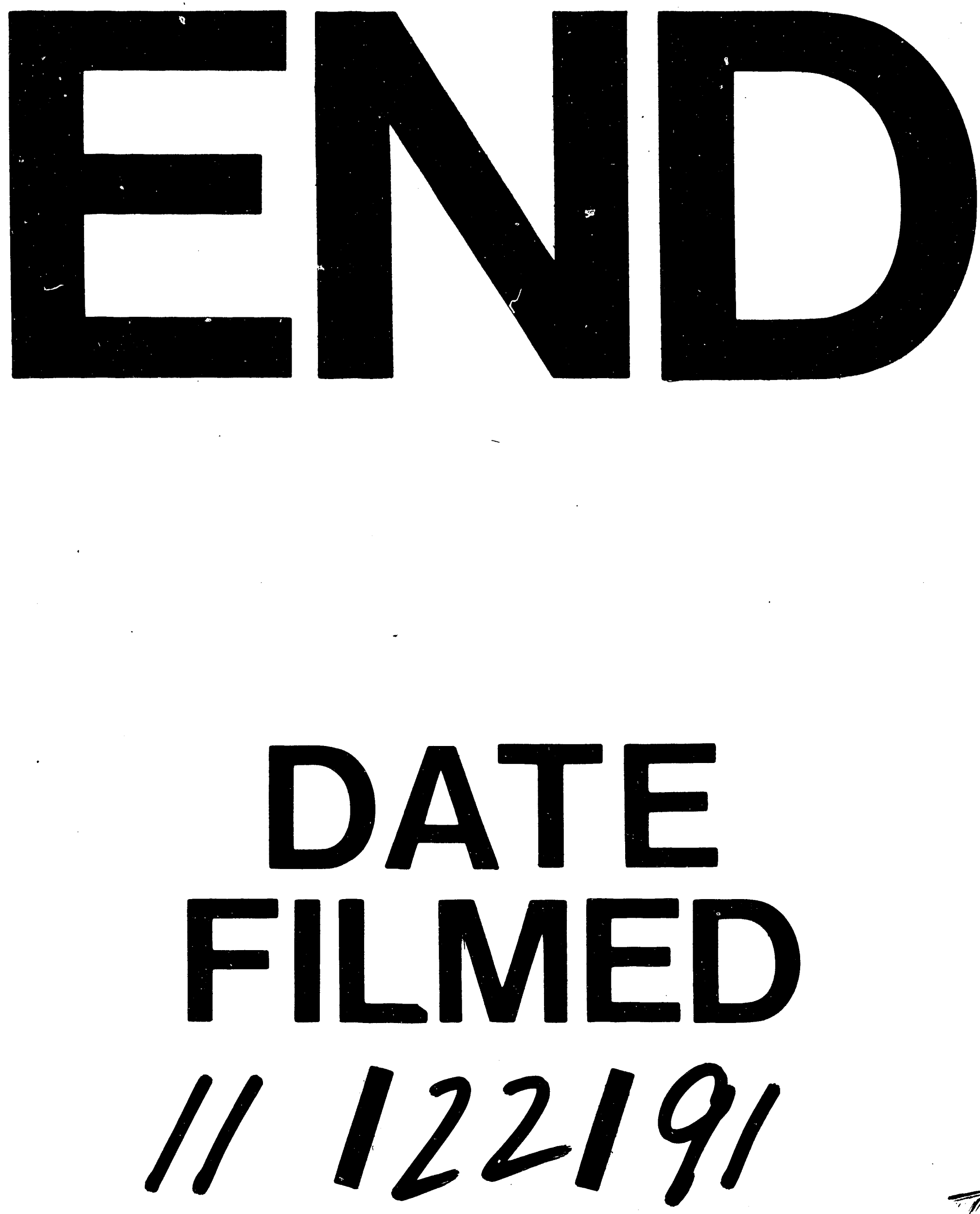
